\title{
Association of Vitamin E Levels with Metabolic Syndrome, and MRI-Derived Body Fat Volumes and Liver Fat Content
}

\author{
Sabina Waniek ${ }^{1}$ (D), Romina di Giuseppe ${ }^{1}$ (D), Sandra Plachta-Danielzik ${ }^{1}$, Ilka Ratjen ${ }^{1}$, \\ Gunnar Jacobs ${ }^{1,2}$, Manja Koch ${ }^{1,3}$, Jan Borggrefe ${ }^{4}$ (D), Marcus Both ${ }^{5}$, Hans-Peter Müller ${ }^{6}$, \\ Jan Kassubek ${ }^{6}$, Ute Nöthlings ${ }^{7}$, Tuba Esatbeyoglu ${ }^{8}$, Sabrina Schlesinger ${ }^{9}$, Gerald Rimbach ${ }^{8}$ \\ and Wolfgang Lieb $1,2, *$ \\ 1 Institute of Epidemiology, Christian-Albrechts University of Kiel, 24105 Kiel, Germany; \\ sabina.waniek@epi.uni-kiel.de (S.W.); romina.digiuseppe@epi.uni-kiel.de (R.d.G.); \\ sandra.plachta-danielzik@epi.uni-kiel.de (S.P.-D.); ilka.ratjen@epi.uni-kiel.de (I.R.); jacobs@popgen.de (G.J.); \\ mkoch@hsph.harvard.edu (M.K.) \\ 2 Biobank PopGen, University Hospital Schleswig-Holstein, Campus Kiel, 24105 Kiel, Germany \\ 3 Department of Nutrition, Harvard T.H. Chan School of Public Health, Boston, MA 02115, USA \\ 4 Institute of Diagnostic and Interventional Radiology, University Hospital Cologne, 50937 Cologne, Germany; \\ jan.borggrefe@uk-koeln.de \\ 5 Department of Radiology and Neuroradiology, University Hospital Schleswig-Holstein, Campus Kiel, \\ 24105 Kiel, Germany; Marcus.both@uksh.de \\ 6 Department of Neurology, University of Ulm, 89081 Ulm, Germany; \\ hans-peter.mueller@uni-ulm.de (H.-P.M.); jan.kassubek@uni-ulm.de (J.K.) \\ 7 Department of Nutrition and Food Science, University of Bonn, 53113 Bonn, Germany; noethlings@uni-bonn.de \\ 8 Institute of Human Nutrition and Food Science, Christian-Albrechts University of Kiel, 24118 Kiel, Germany; \\ tuba.esatbeyoglu@mri.bund.de (T.E.); rimbach@foodsci.uni-kiel.de (G.R.) \\ 9 Institute for Biometrics and Epidemiology, German Diabetes Center (DDZ) at Heinrich Heine University \\ Düsseldorf, 40225 Düsseldorf, Germany; sabrina.schlesinger@DDZ.uni-duesseldorf.de \\ * Correspondence: wolfgang.lieb@epi.uni-kiel.de; Tel.: +49-431-500-30200; Fax: +49-431-500-30204
}

Received: 21 July 2017; Accepted: 11 October 2017; Published: 18 October 2017

\begin{abstract}
We aimed to relate circulating $\alpha$ - and $\gamma$-tocopherol levels to a broad spectrum of adiposity-related traits in a cross-sectional Northern German study. Anthropometric measures were obtained, and adipose tissue volumes and liver fat were quantified by magnetic resonance imaging in 641 individuals (mean age 61 years; $40.6 \%$ women). Concentrations of $\alpha$ - and $\gamma$-tocopherol were measured using high performance liquid chromatography. Multivariable-adjusted linear and logistic regression were used to assess associations of circulating $\alpha$ - and $\gamma$-tocopherol/cholesterol ratio levels with visceral (VAT) and subcutaneous adipose tissue (SAT), liver signal intensity (LSI), fatty liver disease (FLD), metabolic syndrome (MetS), and its individual components. The $\alpha$-tocopherol/cholesterol ratio was positively associated with VAT ( $\beta$ scaled by interquartile range (IQR): 0.036; 95\%Confidence Interval (CI): 0.0003; 0.071) and MetS (Odds Ratio (OR): 1.83; 95\% CI: $1.21-2.76$ for 3rd vs. 1st tertile), and the $\gamma$-tocopherol/cholesterol ratio was positively associated with VAT ( $\beta$ scaled by IQR: $0.066 ; 95 \%$ CI: 0.027; 0.104$)$, SAT ( $\beta$ scaled by IQR: 0.048 ; 95\% CI: 0.010; 0.087) and MetS (OR: 1.87; 95\% CI: 1.23-2.84 for 3rd vs. 1st tertile). $\alpha$ - and $\gamma$-tocopherol levels were positively associated with high triglycerides and low high density lipoprotein cholesterol levels (all $\mathrm{P}_{\text {trend }}<0.05$ ). No association of $\alpha$ - and $\gamma$-tocopherol/cholesterol ratio with LSI/FLD was observed. Circulating vitamin E levels displayed strong associations with VAT and MetS. These observations lay the ground for further investigation in longitudinal studies.
\end{abstract}

Keywords: vitamin E; $\alpha$ - and $\gamma$-tocopherol; metabolic syndrome; body fat volumes; liver fat content 


\section{Introduction}

Metabolic conditions like metabolic syndrome (MetS), fatty liver disease (FLD), and obesity have been linked to increased inflammation and oxidative stress [1-3]. Vitamin E is a lipid-soluble vitamin, encompassing different tocopherols $\left(\alpha-, \beta-, \gamma_{-}^{-}\right.$, and $\delta$-tocopherol) with important anti-oxidative and potentially anti-inflammatory functions [4,5]. In a recent randomized trial [6], vitamin $\mathrm{E}$ administration over 96 weeks performed better than pioglitazone and better than placebo in patients with non-alcoholic steatohepatitis (NASH). It is, therefore, conceivable that vitamin E levels are altered in patients with MetS or FLD and that vitamin E levels are correlated with other adiposity-related traits.

Previous clinical and epidemiologic studies on the association of circulating vitamin E levels with different anthropometric adiposity measures (e.g., waist circumference, body mass index (BMI)) [7-11], the MetS [12-16], and NASH [17-19] produced partially conflicting results. Among the different fat depots, subcutaneous (SAT) and, particularly, visceral adipose tissue (VAT) are considered relevant for metabolic conditions, such as MetS. Whether vitamin E levels are associated with MetS, FLD and other adiposity measures, including SAT and VAT, as determined by magnetic resonance imaging (MRI), is unknown.

Therefore, we aimed to relate circulating levels of $\alpha$ - and $\gamma$-tocopherol to a broad spectrum of adiposity-related traits in a community-based sample from Northern Germany. Specifically, we assessed the associations of plasma $\alpha$ - and $\gamma$-tocopherol levels with MetS and its individual components, MRI-determined VAT, SAT, and liver signal intensity (LSI), as well as with the presence or absence of FLD. We hypothesize that vitamin E levels are altered in individuals with MetS and that vitamin E levels are associated with VAT, SAT, and liver fat, as determined by MRI.

\section{Materials and Methods}

\subsection{Study Sample}

Between 2005 and 2007, a total of 1316 individuals from Northern Germany were recruited by the PopGen biobank [20]. Specifically, the sample consisted of 747 individuals who were identified through official population registries and from 569 blood donors. The first follow-up examination, conducted between 2010 and 2012, was attended by 952 individuals, who received a physical examination conducted by trained personnel and provided blood and urine samples. Furthermore, all participants filled-in a standardized questionnaire on demographic and health-related characteristics (including dietary intake, education, smoking status, and physical activity) and medical history [20,21]. A subsample of participants $(n=641)$ agreed to undergo whole-body MRI. From these participants concentrations of circulating $\alpha$ - and $\gamma$-tocopherol levels were measured. Thus, the association between plasma vitamin E concentrations and MetS was investigated in 641 individuals. A total of 91 individuals had to be excluded from the analyses related to MRI phenotypes because of insufficient imaging quality $(n=35)$, non-adherence to the MRI breathing protocol $(n=40)$, and missing information on quality of MRI assessment $(n=16)$. Further, individuals with self-reported liver disease (hepatitis A, B, C, or D virus infection, hemochromatosis, autoimmune liver disease, or liver cirrhosis $(n=29))$ were excluded. Thus, the association between circulating vitamin E biomarkers and VAT and SAT was assessed in 591 individuals and the association with liver fat and FLD was evaluated in 571 individuals.

The study has been approved by the Ethics Committee of the Medical Faculty of the Christian-Albrechts University Kiel. Written informed consent was obtained from all study participants.

\subsection{Physical Examination and Standardized Questionnaires}

Weight and height were measured with the participant wearing light clothing and no shoes, and $2.0 \mathrm{~kg}$ were subtracted to correct for the remaining clothes. BMI was calculated as body weight $(\mathrm{kg}) /$ height $\left(\mathrm{m}^{2}\right)$. Waist circumference was measured at the midpoint between the lower ribs and iliac crest on the anterior axillary line in a resting expiratory position. After the participants had rested $5 \mathrm{~min}$ in a sitting position, blood pressure was measured twice ( $2 \mathrm{~min}$ interval) using a 
sphygmomanometer [22]. Dietary intake, including information on alcohol consumption during the last 12 months, was assessed by a self-administered semi-quantitative 112-item food-frequency questionnaire (FFQ) designed and validated especially for the German population [23]. The German Food Code and Nutrient Data Base (version II.3) was used to determine energy intake and was provided by the Department of Epidemiology of the German Institute of Human Nutrition Potsdam Rehbrücke [24]. Participants were asked to report their use of vitamin E supplements in the FFQ.

\subsection{Assessment of SAT, VAT, and Liver Fat Using MRI}

Liver fat and adipose tissue (AT) volumes (defined as VAT and SAT) were measured by MRI using a 1.5-T scanner (Magnetom Avanto; Siemens Medical solution, Erlangen, Germany), as described in detail elsewhere [25-27]. VAT was determined as the sum of VAT voxels from the top of the liver to the femoral heads inside the abdominal muscular wall as anatomical border and SAT was determined as the sum of AT voxels underneath the skin layer surrounding the abdomen from the top of the liver to the femur heads. To obtain the volumes (in $\left.\mathrm{dm}^{3}\right)$ of VAT and SAT the voxel size $\left(3.9 \times 2 \times 8 \mathrm{~mm}^{3}\right)$ was multiplied by the number of voxels [26].

Liver fat was quantified as relative LSI difference of the liver on out-of-phase compared with in-phase images in arbitrary units. Both in- and out- of phase images were acquired during a breath hold by using axial T1-weighted gradient echo sequences. Signal intensities were obtained by measuring the average of three circular regions of interest in the liver parenchyma [27].

\subsection{Definitions}

Hypertension was defined as systolic blood pressure $\geq 140 \mathrm{mmHg}$ or diastolic blood pressure $\geq 90 \mathrm{mmHg}$, or self-reported hypertension history or use of antihypertensive medication.

MetS was defined according to the harmonized criteria [28] and was considered present when at least three of the following five criteria were met: (1) elevated triglyceride concentration $(\geq 150 \mathrm{mg} / \mathrm{dL}$ ); (2) reduced high density lipoprotein (HDL)-concentration ( $<40 \mathrm{mg} / \mathrm{dL}$ in men or $<50 \mathrm{mg} / \mathrm{dL}$ in women); (3) elevated blood pressure (systolic blood pressure $\geq 130 \mathrm{mmHg}$ and/or diastolic blood pressure $\geq 85 \mathrm{mmHg}$ or anti-hypertensive treatment); (4) dysglycaemia, defined as elevated plasma fasting glucose $(\geq 100 \mathrm{mg} / \mathrm{dL})$ or anti-diabetic treatment; and (5) abdominal obesity (waist circumference $\geq 94 \mathrm{~cm}$ for men and $\geq 80 \mathrm{~cm}$ for women). In the present definition, information about triglyceride-lowering and HDL-increasing medications were not included because this information was not available in detail in our sample. Type 2 diabetes was defined as glycated hemoglobin $(\mathrm{HbA} 1 \mathrm{c}) \geq 6.5 \%(48 \mathrm{mmol} / \mathrm{mol})$ or fasting glucose $\geq 126 \mathrm{mg} / \mathrm{dL}$, or use of anti-diabetic medication or self-report physician diagnosis. FLD was defined as log liver signal intensity $\geq 3.0$ according to a cut-off, which corresponds to the maximum Youden Index and was derived using spectroscopically determined FLD (liver fat $\geq 5.56 \%$ ) as the reference method [27,29].

Total physical activity was defined as the reported frequency (hour/week) of different activities (leisure and working-time) [30], multiplied by the corresponding metabolic equivalent (MET)-value, and summed up for all activities [22,31]. Participants were classified into 3 categories to determine smoking status: no-smokers if they had never smoked; former smokers if they had smoked in the past and quit smoking more than 1 year ago; and current smokers if they were currently smoking 1 or more cigarettes per day. Participants were categorized according to the level of education into three categories: low ( $\leq 9$ years), middle (10 years), or high ( $\geq 11$ years).

\subsection{Laboratory Analyses}

Fasting blood (EDTA whole-blood and lithium heparin) samples were obtained from participants in a sitting position. All blood samples were centrifuged, aliquoted, and stored at $-80^{\circ} \mathrm{C}$. In fresh blood samples, concentrations of C-reactive protein (CRP), triglycerides, HDL-cholesterol, and total cholesterol were analyzed by enzymatic colorimetry (Roche Diagnostic, Mannheim, Germany); the concentration of glucose was determined by using enzymatic ultraviolet tests (Roche Diagnostic, 
Mannheim, Germany), and $\mathrm{HbA1c}$ concentrations were determined by using high performance liquid chromatography (HPLC) and photometric detection (Bio-Rad Laboratories, Munich, Germany) in EDTA plasma.

Laboratory blood analyses were performed in the laboratory for clinical chemistry of the University Hospital Schleswig-Holstein, Campus Kiel in Germany.

The Institute of Human Nutrition and Food Science at the Christian-Albrechts-University of Kiel in Germany measured plasma vitamin E ( $\alpha$ - and $\gamma$-tocopherol) levels using a HPLC with fluorescence detection. An external standard curve was used to quantify vitamin E concentrations using a Jasco HPLC system (Jasco GmbH Deutschland, Gross-Umstadt, Germany; equipped with an autosampler (Jasco AS-2057), pump (PU-2080), ternary gradient unit (LG-2080-02), 3 line degasser (DG-2080-53), and fluorescence detector (FP2020 Plus) $)$ with a Waters Spherisorb ODS-2,3 $\mu \mathrm{m}$ column $(100 \times 4.6 \mathrm{~mm})$ using methanol:water $(98: 2, v / v)$ as mobile phase. The fluorescence detector operated an excitation wavelength of $290 \mathrm{~nm}$ and emission wavelength of $325 \mathrm{~nm}$. The flow rate of the mobile phase was set at $1.2 \mathrm{~mL} / \mathrm{min}$. Duplicate measurements were performed and the injection volume was set at $40 \mu \mathrm{L}$. Plasma ( $50 \mu \mathrm{L}$ ) was homogenized in $2 \mathrm{~mL} \mathrm{1 \%}$ ascorbic acid (in ethanol), $700 \mu \mathrm{L}$ deionised water, $50 \mu \mathrm{L}$ $0.1 \%$ butylated hydroxytoluol (in ethanol), and $2 \mathrm{~mL}$-hexane were prepared for analysing the samples. The samples were centrifuged $\left(1000 \times g\right.$ for $5 \mathrm{~min}$ at $\left.4{ }^{\circ} \mathrm{C}\right)$. After separating the phases, $1000 \mu \mathrm{L}$ of the upper phase was dried under vacuum in a RC-1010 centrifugal evaporator (Jouan, Saint-Herblain, France). The samples were re-suspended in $200 \mu \mathrm{L}$ mobile phase (methanol:water, 98:2, v/v) [32]. The coefficients of variation for $\alpha$ - and $\gamma$-tocopherol were $1.05 \%$ and $1.29 \%$, respectively.

\subsection{Statistical Analyses}

Some few missing values of covariates were replaced by a simple imputation, as follows: When values of categorical variables were missing, they were replaced by the most commonly observed category of that respective variable $(n=10)$. Normally distributed continuous missing variables were imputed by the respective mean and skewed variables by the sex-specific median $(n=2)$. Detailed information of missing covariates are provided in Supplementary Materials Table S1.

CRP values below $0.9 \mathrm{mg} / \mathrm{dL}$ (detection limit) were assigned a value equal to the half of the detection limit $(n=247)$. Values of $\gamma$-tocopherol $(n=14$, respectively) were imputed by the lowest $\gamma$-tocopherol concentration measured in our sample. Detailed information of missing covariates are provided in Supplementary Materials Table S1.

Because vitamin E is bound to lipoproteins in the blood stream [33], cholesterol-adjusted $\alpha$ - and $\gamma$-tocopherol levels $(\mu \mathrm{mol} / \mathrm{mmol})$ were calculated by dividing $\alpha$ - and $\gamma$-tocopherol concentrations $(\mu \mathrm{mol} / \mathrm{L})$ by total cholesterol $(\mathrm{mmol} / \mathrm{L})[34]$.

We performed the following analyses: For descriptive purposes, anthropometric, lifestyle, and clinical factors were compared across tertiles of the $\alpha$ - and $\gamma$-tocopherol/cholesterol ratios. Differences in median of continuous variables were tested by using Wilcoxon s rank-sum test, and differences in categorical variables were assessed by using a chi-square test.

Restricted cubic splines analyses displayed linear associations between vitamin E biomarkers and continuous (VAT, SAT, LSI) and binary (MetS, and FLD) outcomes. Third, linear and logistic regression models were used to relate circulating vitamin $\mathrm{E}$ ( $\alpha$ - and $\gamma$-tocopherol/cholesterol ratio, each biomarker considered separately) levels to continuous outcomes (VAT, SAT, LSI) and binary outcomes (MetS, individual components of MetS, FLD), respectively. In linear regression models, both $\alpha$ - and $\gamma$-tocopherol levels were scaled to their interquartile range (IQR) and $\beta$ coefficients interpreted as comparing VAT, SAT, and LSI values of a person with a typical "high" $\alpha$ - or $\gamma$-tocopherol value to a person with a typical "low" value.

Adjusted means of VAT, SAT, and LSI were calculated by general linear models, respectively. We ran age- and sex-adjusted, as well as multivariable-adjusted, models which included, based on literature research [12,13], age (continuous in years) and sex, education (low, medium, high), physical activity (continuous in MET-hour/week), smoking status (never, current, former), vitamin E 
supplementation (yes, no), alcohol intake (continuous in g/day), and total energy intake (continuous in $\mathrm{kJ} /$ day) as potential confounders. Furthermore, the models with continuous VAT and SAT as outcome variables were additionally adjusted for BMI (continuous in $\mathrm{kg} / \mathrm{m}^{2}$ ) to assess whether VAT and SAT were associated with circulating vitamin E levels independent of BMI. Individual components of MetS were adjusted for each of the other four criteria for the MetS.

Potential interactions of age, sex, and vitamin E supplementation with each metabolic outcome (VAT, SAT, LSI, MetS, individual components of MetS, FLD) were tested by including multiplicative interaction terms into the regression models. In a sensitivity analysis, we excluded vitamin $\mathrm{E}$ supplement users and we related $\alpha$ - and $\gamma$-tocopherol/cholesterol ratio levels (each biomarker considered separately) to each selected metabolic outcome (Supplementary Materials Tables S4-S6). Furthermore, we excluded individuals who reported a consumption of alcohol more than $20 \mathrm{~g} /$ day $(n=134)$ when examining the association of $\alpha$ - and $\gamma$-tocopherol/cholesterol ratio with FLD.

Categorical variables with more than two categories were included as indicator variables. $P_{\text {trend }}$ was calculated across tertiles using median values of $\alpha$ - and $\gamma$-tocopherol/cholesterol ratio within each tertile and we used these values as continuous variables.

All statistical tests were two-sided and considered to be significant when $p$ values $<0.05$. All analyses were performed with SAS 9.4 (SAS Institute, Cary, NC, USA).

\section{Results}

\subsection{General Characteristics}

General characteristics of the study sample according to tertiles of the $\alpha$ - and $\gamma$-tocopherol/ cholesterol ratio are depicted in Tables 1 and 2, respectively. Triglycerides levels were higher in the 3rd tertile compared to the 1st tertile of the $\alpha$-tocopherol/cholesterol ratio. Furthermore, the proportion of individuals with MetS was higher in the upper tertiles of the $\alpha$-tocopherol/cholesterol ratio. Similarly, triglycerides levels were higher in the 3rd tertile compared to the 1st tertile of the $\gamma$-tocopherol/cholesterol ratio. Furthermore, BMI and waist circumference, CRP levels, as well as VAT and SAT were higher in the 3rd tertile compared to the 1st tertile of the $\gamma$-tocopherol/cholesterol ratio. In addition, the prevalence of the MetS and of diabetes rose with tertiles of the $\gamma$-tocopherol/cholesterol ratio. The proportion of vitamin E supplement users was highest in the bottom tertile of $\gamma$-tocopherol/cholesterol ratio.

Table 1. General characteristics of the PopGen control study population ( $n=641)$ according to tertiles (T) of $\alpha$-tocopherol/cholesterol ratio.

\begin{tabular}{|c|c|c|c|c|c|c|c|}
\hline \multirow{2}{*}{ Characteristics } & \multicolumn{6}{|c|}{ Tertiles $\alpha$-Tocopherol/Cholesterol Ratio } & \multirow{2}{*}{$p$} \\
\hline & \multicolumn{2}{|c|}{ T1 $(n=213)$} & \multicolumn{2}{|c|}{$\mathrm{T} 2(n=214)$} & \multicolumn{2}{|c|}{ T3 $(n=214)$} & \\
\hline $\begin{array}{l}\alpha \text {-tocopherol/cholesterol ratio } \\
\text { (IQR), } \mu \mathrm{mol} / \mathrm{mmol}\end{array}$ & 4.63 & $(4.25-4.88)$ & 5.53 & $(5.36-5.72)$ & 6.74 & $(6.33-7.59)$ & \\
\hline Men, $\%$ & 55.9 & & 61.7 & & 60.8 & & 0.422 \\
\hline Age, years & 63.0 & $(56.0-70.0)$ & 61.5 & $(54.0-71.0)$ & 62.0 & $(51.0-71.0)$ & 0.411 \\
\hline Body mass index, $\mathrm{kg} / \mathrm{m}^{2}$ & 26.6 & $(23.3-29.8)$ & 26.7 & $(24.8-29.4)$ & 26.7 & $(24.6-29.2)$ & 0.633 \\
\hline Waist circumference, $\mathrm{cm}$ & & & & & & & \\
\hline Men & 100.0 & $(92.8-107.4)$ & 100.2 & $(92.7-105.9)$ & 99.4 & $(93.5-106.8)$ & 0.956 \\
\hline Women & 87.1 & $(78.5-96.4)$ & 88.5 & $(83.2-97.4)$ & 92.4 & $(80.2-99.6)$ & 0.199 \\
\hline
\end{tabular}


Table 1. Cont.

\begin{tabular}{|c|c|c|c|c|c|c|c|}
\hline \multirow{3}{*}{$\begin{array}{c}\text { Characteristics } \\
\text { Systolic blood pressure, } \mathrm{mm} / \mathrm{Hg}\end{array}$} & \multicolumn{6}{|c|}{ Tertiles $\alpha$-Tocopherol/Cholesterol Ratio } & \multirow{3}{*}{$\begin{array}{c}p \\
0.856\end{array}$} \\
\hline & \multicolumn{2}{|c|}{ T1 $(n=213)$} & \multicolumn{2}{|c|}{$\mathrm{T} 2(n=214)$} & \multicolumn{2}{|c|}{ T3 $(n=214)$} & \\
\hline & 139.0 & $(127.5-150.0)$ & 140.0 & $(125.0-150.0)$ & 138.3 & $(125.0-150.0)$ & \\
\hline $\begin{array}{c}\text { Diastolic blood pressure, } \\
\mathrm{mm} / \mathrm{Hg}\end{array}$ & 85.0 & $(80.0-90.0)$ & 85.0 & $(80.0-90.0)$ & 82.3 & $(80.0-90.0)$ & 0.341 \\
\hline Prevalent hypertension, $\%$ & 68.1 & & 71.0 & & 67.8 & & 0.723 \\
\hline Current smokers, $\%$ & 10.1 & & 9.8 & & 12.2 & & 0.640 \\
\hline $\begin{array}{l}\text { Physical activity, } \\
\text { MET-hour/week }\end{array}$ & 98.3 & $(61.5-141.6)$ & 84.2 & $(54.8-120.1))$ & 90.0 & $(59.3-131.7)$ & 0.074 \\
\hline High education ( $\geq 11$ years), $\%$ & 29.1 & & 40.7 & & 37.9 & & 0.143 \\
\hline Alcohol consumption, g/day & 8.67 & $(2.76-17.0)$ & 8.58 & $(4.09-17.95)$ & 10.96 & $(4.15-20.05)$ & 0.114 \\
\hline Vitamin E supplementation, \% & 5.6 & & 6.5 & & 10.3 & & 0.154 \\
\hline Prevalent diabetes, $\%$ & 8.9 & & 8.9 & & 14.5 & & 0.099 \\
\hline Metabolic syndrome, \% & 36.6 & & 36.0 & & 48.1 & & 0.016 \\
\hline C-reactive protein, $\mathrm{mg} / \mathrm{dL}$ & 1.10 & $(0.45-2.60)$ & 1.20 & $(0.45-2.40)$ & 1.40 & $(0.45-2.20)$ & 0.531 \\
\hline HDL-cholesterol, mg/dL & 67.0 & $(56.0-82.0)$ & 63.5 & $(54.0-76.0)$ & 57.5 & $(49.0-72.0)$ & $<0.0001$ \\
\hline Triglycerides, mg/dL & 96.0 & $(71.0-123.0)$ & 104.0 & $(78.0-132.0)$ & 123.0 & $(84.0-169.0)$ & $<0.0001$ \\
\hline Diabetes medication, \% * & 3.6 & & 7.1 & & 14.6 & & 0.015 \\
\hline Lipid-lowering medication, $\%$ * & 13.6 & & 29.3 & & 45.8 & & $<0.0001$ \\
\hline Fatty liver disease, $\%{ }^{\dagger}$ & 38.9 & & 38.5 & & 40.3 & & 0.928 \\
\hline Liver signal intensity $^{\dagger}$ & 18.6 & $(14.9-23.4)$ & 18.2 & $(15.0-22.1)$ & 18.0 & $(14.5-24.7)$ & 0.925 \\
\hline Visceral adipose tissue, $\mathrm{dm}^{3} \ddagger$ & 3.70 & $(2.18-5.02)$ & 3.90 & $(2.41-5.25)$ & 3.94 & $(2.54-5.37)$ & 0.478 \\
\hline $\begin{array}{c}\text { Subcutaneous adipose } \\
\text { tissue, } \mathrm{dm}^{3} \ddagger\end{array}$ & 5.91 & $(4.45-8.23)$ & 6.45 & $(4.75-8.53)$ & 6.10 & $(4.88-8.24)$ & 0.546 \\
\hline
\end{tabular}

Data are reported as percentages (\%) or median and interquartile range (IQR). ${ }^{*} n=305,{ }^{\dagger} n=571,{ }^{\ddagger} n=591$; MET: Metabolic equivalent; HDL: High density lipoprotein

Table 2. General characteristics of the PopGen control study population $(n=641)$ according to tertiles of $\gamma$-tocopherol/cholesterol ratio.

\begin{tabular}{|c|c|c|c|c|c|c|c|}
\hline \multirow{2}{*}{ Characteristics } & \multicolumn{6}{|c|}{ Tertiles (T) $\gamma$-Tocopherol/Cholesterol Ratio } & \multirow{2}{*}{$p$} \\
\hline & \multicolumn{2}{|c|}{$\mathrm{T} 1(n=213)$} & \multicolumn{2}{|c|}{$\mathrm{T} 2(n=214)$} & \multicolumn{2}{|c|}{ T3 $(n=214)$} & \\
\hline $\begin{array}{l}\text { Median } \gamma \text {-tocopherol/cholesterol } \\
\text { ratio (IQR), } \mu \mathrm{mol} / \mathrm{mmol}\end{array}$ & 0.16 & $(0.13-0.18)$ & 0.24 & $(0.22-0.26)$ & 0.35 & $(0.31-0.42)$ & \\
\hline Men, $\%$ & 60.06 & & 57.9 & & 59.8 & & 0.851 \\
\hline Age, years & 63.0 & $(55.0-71.0)$ & 61.5 & $(55.0-71.0)$ & 62.0 & $(54.0-69.0)$ & 0.709 \\
\hline Body mass index, $\mathrm{kg} / \mathrm{m} 2$ & 26.1 & $(23.4-28.9)$ & 27.3 & $(24.8-29.6)$ & 26.8 & $(24.9-30.7)$ & 0.005 \\
\hline Women & 85.3 & $(77.4-93.6)$ & 89.0 & $(82.4-98.0)$ & 91.8 & $(80.2-103.5)$ & 0.002 \\
\hline Systolic blood pressure, $\mathrm{mm} / \mathrm{Hg}$ & 139.0 & $(126.5-150.0)$ & 140.0 & $(125.0-150.0)$ & 139.0 & $(127.5-150.0)$ & 0.858 \\
\hline $\begin{array}{c}\text { Diastolic blood pressure, } \\
\mathrm{mm} / \mathrm{Hg}\end{array}$ & 85.0 & $(80.0-90.0)$ & 85.0 & $(80.0-90.0)$ & 85.0 & $(80.0-90.0)$ & 0.853 \\
\hline Prevalent hypertension, $\%$ & 67.1 & & 71.5 & & 68.2 & & 0.598 \\
\hline Alcohol consumption, g/d & 8.87 & $(3.20-16.79)$ & 10.18 & $(3.82-20.3)$ & 9.74 & $(4.0-20.13)$ & 0.504 \\
\hline Vitamin E supplementation, $\%$ & 14.6 & & 2.8 & & 5.1 & & $<0.0001$ \\
\hline Prevalent diabetes, $\%$ & 8.0 & & 8.9 & & 15.4 & & 0.026 \\
\hline Metabolic syndrome, \% & 32.9 & & 41.6 & & 46.3 & & 0.017 \\
\hline C-reactive protein, $\mathrm{mg} / \mathrm{dL}$ & 1.0 & $(0.45-1.90)$ & 1.30 & $(0.45-2.80)$ & 1.40 & $(0.45-2.60)$ & 0.009 \\
\hline HDL-cholesterol, mg/dL & 66.0 & $(54.0-79.0)$ & 62.0 & $(53.0-79.0)$ & 60.0 & $(51.0-74.0)$ & 0.023 \\
\hline Triglycerides, mg/dL & 100.0 & $(76.0-131.0)$ & 103.0 & $(72.0-133.0)$ & 115.5 & $(80.0-158.0)$ & 0.004 \\
\hline Diabetes medication, $\%$ * & 4.0 & & 3.9 & & 17.0 & & 0.0005 \\
\hline Lipid-lowering medication, $\%$ * & 22.8 & & 26.9 & & 37.0 & & 0.073 \\
\hline Fatty liver disease, $\%^{+}$ & 37.4 & & 36.7 & & 43.7 & & 0.303 \\
\hline Liver signal intensity $^{\dagger}$ & 18.5 & $(14.7-22.4)$ & 17.9 & $(14.5-24.1)$ & 18.8 & $(14.8-24.2)$ & 0.599 \\
\hline Visceral adipose tissue, $\mathrm{dm}^{3} \ddagger$ & 3.55 & $(2.26-4.95)$ & 3.82 & $(2.46-5.16)$ & 4.15 & $(2.71-5.77)$ & 0.013 \\
\hline
\end{tabular}

Data are reported as percentages (\%) or median and interquartile range (IQR). ${ }^{*} n=305,{ }^{\dagger} n=571,{ }^{\ddagger} n=591$; MET:

Metabolic equivalent; HDL: High density lipoprotein. 


\subsection{Association of $\alpha$-Tocopherol/Cholesterol Ratio with Metabolic Traits}

In multivariable-adjusted linear regression models, plasma $\alpha$-tocopherol/cholesterol ratio displayed a statistically significant association with VAT ( $\beta$ scaled by IQR: 0.036 ; $95 \%$ Confidence Interval (CI): $0.0003 ; 0.071)$, including a model additionally adjusted for BMI ( $\beta$ scaled by IQR: 0.026 ; 95\% CI: $0.002 ; 0.050$ ) (Table 3). Furthermore, consistent associations of plasma $\alpha$-tocopherol/cholesterol ratio with the MetS were observed (Odds Ratio (OR): 1.83; 95\% CI: 1.21-2.76 for 3rd vs. 1st tertile; $\left.\mathrm{P}_{\text {trend }}=0.003\right)($ Table 5$)$, driven by positive associations with high triglycerides (OR: $3.02 ; 95 \%$ CI: $1.80-5.06$ for 3rd vs. 1st tertile; $\mathrm{P}_{\text {trend }}<0.0001$ ) and low HDL-cholesterol levels (OR: 2.52; 95\% CI: $0.97-6.56$ for 3rd vs. 1st tertile; $\mathrm{P}_{\text {trend }}=0.033$ ) (Supplementary Materials Table S2). The $\alpha$-tocopherol/cholesterol ratio was neither associated with SAT, nor with LSI, modeled as a continuous or binary trait (FLD) (Table 3 and Table 5).

Table 3. Multivariable-adjusted means and 95\% CI of VAT, SAT, and LSI according to tertiles of $\alpha$-tocopherol/cholesterol ratio, and scaled by IQR.

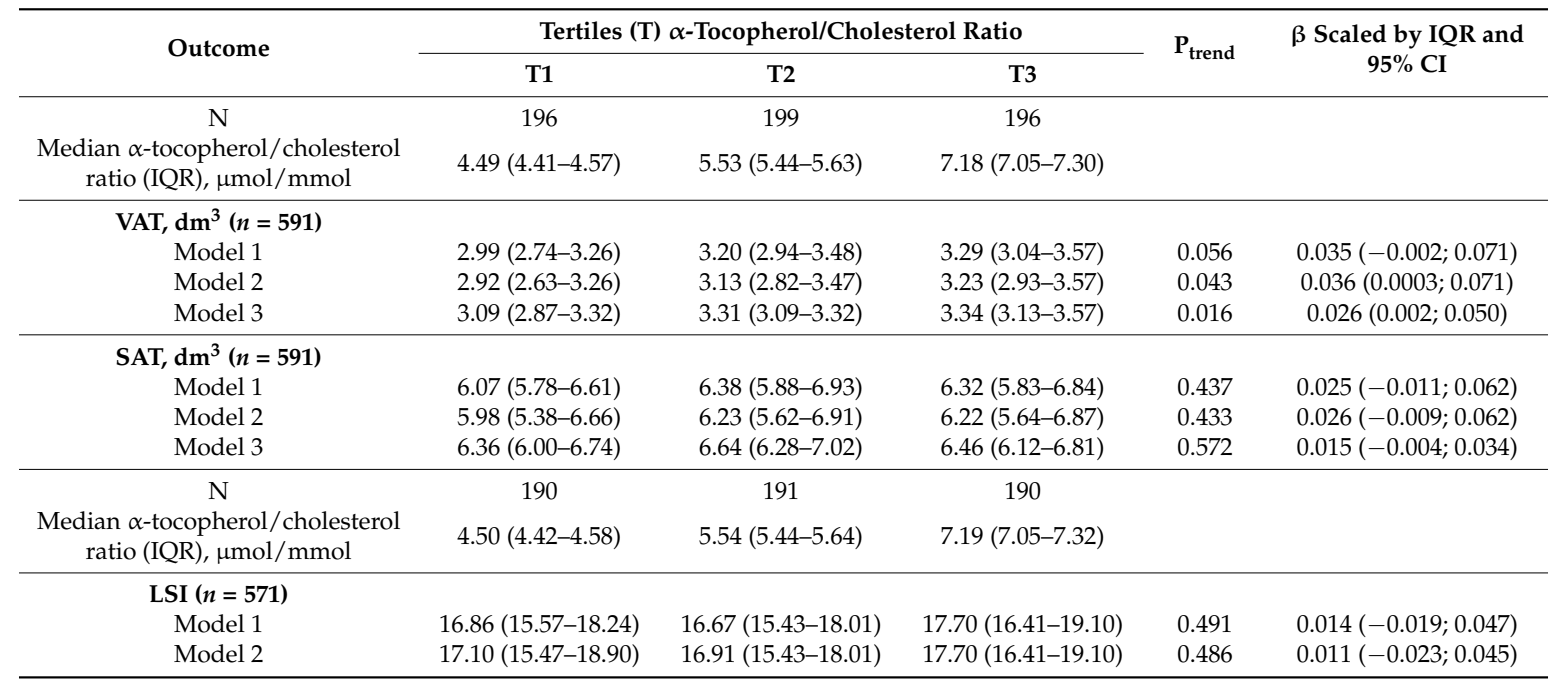

VAT: Visceral adipose tissue; SAT: Subcutaneous adipose tissue; LSI: Liver signal intensity; BMI: Body mass index; IQR: Interquartile range; CI: Confidence Interval. Model 1: Adjusted for age and sex. Model 2 is model 1 but additionally adjusted for education, physical activity, smoking status, vitamin E supplementation, alcohol intake, and total energy intake. Model 3 is model 2 but additionally adjusted for BMI.

\subsection{Association of $\gamma$-Tocopherol/Cholesterol Ratio with Metabolic Traits}

In multivariable-adjusted linear and logistic regression models, plasma $\gamma$-tocopherol/cholesterol ratio showed statistically significant associations with VAT ( $\beta$ scaled by IQR: 0.066 ; $95 \%$ CI: 0.027 ; 0.104), SAT ( $\beta$ scaled by IQR: 0.048; 95\% CI: 0.010; 0.087), and the MetS (OR: 1.87; 95\% CI: $1.23-2.84$ for $3 \mathrm{r}^{\mathrm{d}}$ vs. 1st tertile; $\left.\mathrm{P}_{\text {trend }}=0.004\right)$ (Tables 4 and 5$)$. The association with VAT persisted upon additional adjustment for BMI ( $\beta$ scaled by IQR: $0.037 ; 95 \%$ CI: $0.011 ; 0.063)$, whereas adding BMI to the model rendered the association of $\gamma$-tocopherol/cholesterol ratio with SAT statistically non-significant ( $\beta$ scaled by IQR: $0.015 ; 95 \%$ CI: $-0.006 ; 0.037$ ) (Table 4). Regarding the individual components of the MetS, the $\gamma$-tocopherol/cholesterol ratio was positively related to hypertriglyceridemia (OR: 1.81; 95\% CI: 1.08-3.06 for 3rd vs. 1st tertile; $\mathrm{P}_{\text {trend }}=0.014$ ) and low HDL-cholesterol levels (OR: 4.67; 95\% CI: $1.42-15.41$ for 3rd vs. 1st tertile; $\mathrm{P}_{\text {trend }}=0.018$ ) in multivariable-adjusted models (Supplementary Materials Table S3). 
Table 4. Multivariable-adjusted means and 95\% CI of VAT, SAT, and LSI according to tertiles of $\gamma$-tocopherol/cholesterol ratio, and scaled by IQR.

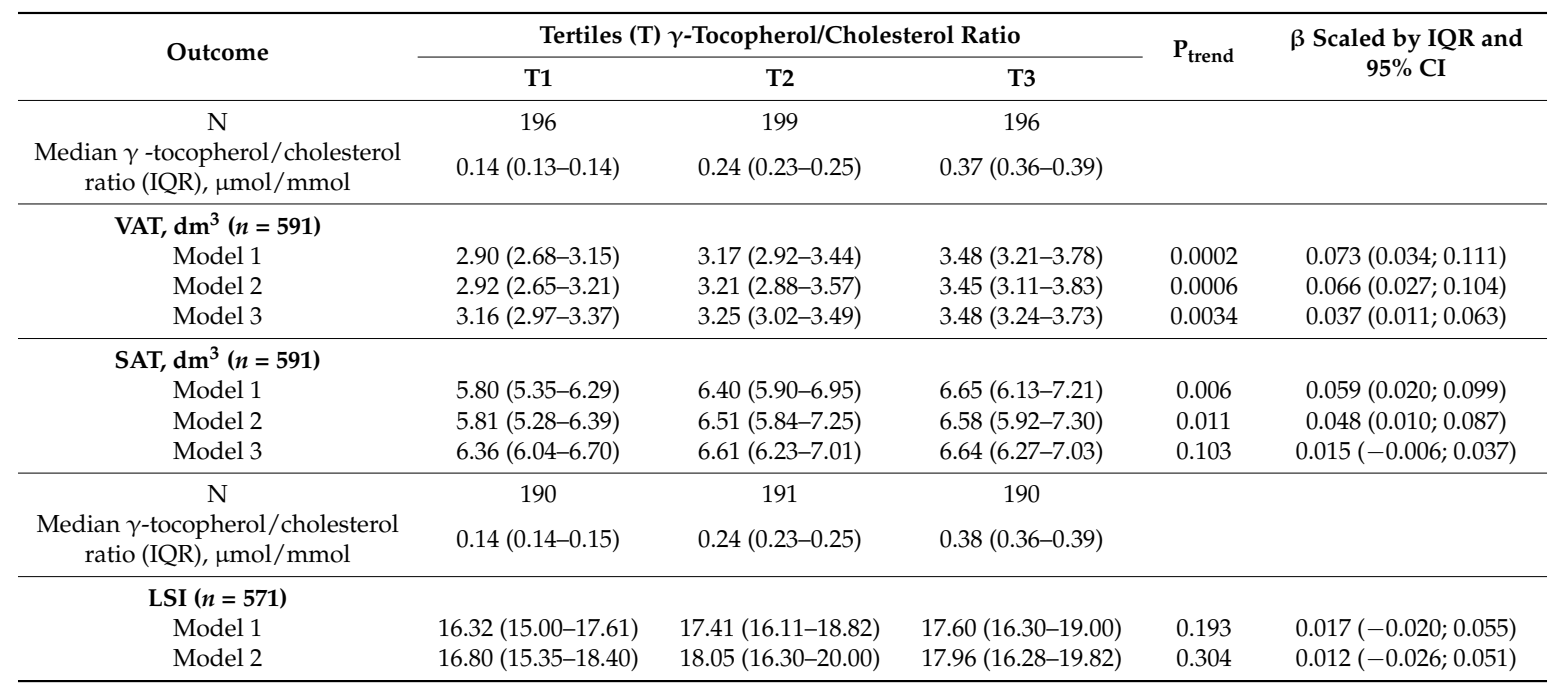

VAT: Visceral adipose tissue; SAT: Subcutaneous adipose tissue; LSI: Liver signal intensity; BMI: Body mass index; IQR: Interquartile range; CI: Confidence interval. Model 1: Adjusted for age and sex. Model 2 is model 1 but additionally adjusted for education, physical activity, smoking status, vitamin E supplementation, alcohol intake, and total energy intake. Model 3 is model 2 but additionally adjusted for BMI.

Table 5. Odds Ratio and 95\% Confidence Interval for the association of $\alpha$ - and $\gamma$-tocopherol/cholesterol ratio with metabolic syndrome (MetS) and fatty liver disease (FLD).

\begin{tabular}{|c|c|c|c|c|}
\hline \multirow{2}{*}{ Outcome } & \multicolumn{3}{|c|}{ Tertiles (T) of $\alpha$-Tocopherol/Cholesterol Ratio } & \multirow{2}{*}{$\mathbf{P}_{\text {trend }}$} \\
\hline & T1 & T2 & T3 & \\
\hline $\begin{array}{l}\text { Median } \alpha \text {-tocopherol/cholesterol } \\
\text { ratio (IQR), } \mu \mathrm{mol} / \mathrm{mmol}\end{array}$ & $4.63(4.25-4.88)$ & $5.53(5.36-5.72)$ & $6.74(6.33-7.59)$ & \\
\hline MetS (yes/no) $(258 / 383)$ & $(78 / 135)$ & $(77 / 137)$ & $(103 / 111)$ & \\
\hline Model 1 & Reference & $1.01(0.67-1.51)$ & $1.72(1.15-2.58)$ & 0.006 \\
\hline Model 2 & Reference & $1.09(0.72-1.65)$ & $1.83(1.21-2.76)$ & 0.003 \\
\hline $\begin{array}{l}\text { Median } \alpha \text {-tocopherol } / \text { cholesterol } \\
\text { ratio (IQR), } \mu \mathrm{mol} / \mathrm{mmol}\end{array}$ & $4.61(4.25-4.87)$ & $5.52(5.35-5.73)$ & $6.75(6.29-7.57)$ & \\
\hline FLD (yes/no) $(224 / 347)$ & $(72 / 113)$ & $(75 / 120)$ & $(77 / 114)$ & \\
\hline Model 1 & Reference & $1.03(0.67-1.58)$ & $1.11(0.72-1.70)$ & 0.631 \\
\hline \multirow[t]{3}{*}{ Model 2} & Reference & $1.01(0.65-1.55)$ & $1.09(0.70-1.68)$ & 0.694 \\
\hline & \multicolumn{3}{|c|}{ Tertiles (T) of $\gamma$-Tocopherol/Cholesterol Ratio } & \multirow{2}{*}{$\mathbf{P}_{\text {trend }}$} \\
\hline & T1 & T2 & T3 & \\
\hline $\begin{array}{l}\text { Median } \gamma \text {-tocopherol } / \text { cholesterol } \\
\text { ratio (IQR), } \mu \mathrm{mol} / \mathrm{mmol}\end{array}$ & $0.16(0.13-0.18)$ & $0.24(0.22-0.26)$ & $0.35(0.31-0.41)$ & \\
\hline MetS (yes/no) $(258 / 383)$ & $(70 / 143)$ & $(89 / 125)$ & $(99 / 115)$ & \\
\hline Model 1 & Reference & $1.58(1.05-2.39)$ & $1.92(1.28-2.89)$ & 0.002 \\
\hline Model 2 & Reference & $1.50(0.98-2.29)$ & $1.87(1.23-2.84)$ & 0.004 \\
\hline $\begin{array}{l}\text { Median } \gamma \text {-tocopherol/cholesterol } \\
\text { ratio (IQR), } \mu \mathrm{mol} / \mathrm{mmol}\end{array}$ & $0.16(0.13-0.18)$ & $0.24(0.22-0.26)$ & $0.34(0.31-0.42)$ & \\
\hline FLD (yes/no) $(224 / 347)$ & $(72 / 113)$ & $(75 / 120)$ & $(77 / 114)$ & \\
\hline Model 1 & Reference & $0.99(0.65-1.52)$ & $1.38(0.90-2.10)$ & 0.124 \\
\hline Model 2 & Reference & $0.97(0.62-1.51)$ & $1.31(0.85-2.02)$ & 0.204 \\
\hline
\end{tabular}

IQR: Interquartile range; MetS: Metabolic syndrome; FLD: Fatty liver disease model 1: adjusted for age and sex. Model 2 is model 1 but additionally adjusted for education, physical activity, smoking status, vitamin $\mathrm{E}$ supplementation, alcohol intake, and total energy intake.

No association of $\gamma$-tocopherol/cholesterol ratio with LSI (continuous trait) or FLD (binary trait) was observed (Tables 4 and 5). 


\subsection{Assessment of Interactions and Sensitivity Analyses}

No significant interactions between circulating $\alpha$ - and $\gamma$-tocopherol/cholesterol concentrations and age, sex, or vitamin E supplementation in relation to each selected outcome were observed ( $p>0.05$ for all). In a sensitivity analysis, excluding vitamin E supplement users, the magnitude and the direction of the associations were essentially unchanged (Supplementary Materials Tables S4-S6).

With respect of the association of vitamin E levels with FLD, the results were essentially unchanged after excluding individuals with an alcohol consumption of more than $20 \mathrm{~g} /$ day. In multivariable-adjusted models, the $\alpha$ - and $\gamma$-tocopherol/cholesterol ratio were not statistically significantly related to the probability of having FLD (OR: 1.09; 95\% CI: 0.66-1.80 for 3rd vs. 1st tertile; $\mathrm{P}_{\text {trend }}=0.268$ and OR: $1.51 ; 95 \%$ CI: $0.94-2.55$ for 3rd vs. 1 st tertile; $P_{\text {trend }}=0.239$, respectively).

\section{Discussion}

\subsection{Principal Observations}

In a community-based sample from Northern Germany, the $\alpha$-tocopherol/cholesterol ratio and the $\gamma$-tocopherol/cholesterol ratio were positively associated with VAT, SAT, MetS, and its components, high triglycerides and low HDL-cholesterol levels. No significant associations were observed when $\alpha$ and $\gamma$-tocopherol/cholesterol ratios were studied in relation to LSI or FLD.

\subsection{In the Context of the Published Literature}

\subsubsection{Vitamin E Levels and Measures of Adiposity and Adipose Tissue Volumes}

We observed a consistent association of $\alpha$ - and $\gamma$ tocopherol/cholesterol ratio with VAT; the $\gamma$-tocopherol/cholesterol ratio was also associated with SAT. This is in line with several prior studies that reported positive associations of circulating vitamin E levels with adiposity measures (e.g., BMI, waist circumference, waist-to-hip ratio, and waist-to-height ratio) [7-9]. For example, in a subsample of participants in the Women's Health Initiative $(n=2672)$, circulating $\gamma$-tocopherol levels were positively and strongly associated with BMI, waist circumference, and waist-to-height ratio, while $\alpha$-tocopherol levels were only positively associated with waist-to-hip ratio [7]. Likewise, Chai et al. [8] reported in 180 premenopausal women that $\gamma$-tocopherol levels were significantly higher in obese individuals, whereas $\alpha$-tocopherol levels did not differ among BMI subgroups.

With respect to $\alpha$-tocopherol, Wallström et al. [9] reported that serum levels of $\alpha$-tocopherol were positively related to central adiposity (defined as waist circumference and waist-to-hip ratio), but BMI was only associated with $\alpha$-tocopherol in men. Body fat percentage (determined by bioelectrical impedance analysis), however, was not significantly associated with vitamin E [9]. Interestingly, in a subsample of healthy postmenopausal women $(n=48), \alpha$-tocopherol was identified as predictor of MRI-determined total fat [35]. By contrast, in a sample of 580 women, no association of vitamin E levels with measures of adiposity (BMI, waist circumference, waist-to-height ratio, visceral adiposity, and total body fat) determined by dual-energy $\mathrm{x}$-ray absorptiometry was observed [10].

\subsubsection{Vitamin E Levels and the Metabolic Syndrome}

We observed consistent positive associations of $\alpha$ - and $\gamma$-tocopherol/cholesterol ratio levels with MetS in different multivariable-adjusted statistical models. These associations were driven by a positive association with low HDL-cholesterol levels and high triglycerides levels. The association of vitamin E levels with lipid traits is biologically plausible because the lipid-soluble vitamin $\mathrm{E}$ is transported in the blood by lipoproteins [33].

In contrast to our observations, in one study, lower levels of plasma $\alpha$-tocopherol levels were reported in individuals with MetS $(n=182)$ compared to healthy adults $(n=91)$ [14]. In a much larger sample from the 2001-2006 National Health Examination Survey (NHANES; $n=3008$ ), no association of vitamin E levels with MetS was reported. However, in further analyses, the authors observed that 
vitamin E concentrations were significantly positively related to the number of MetS criteria [12], which lends some support to our results.

Regarding individual components of MetS, vitamin E controlled for lipids showed a positive association with hypertriglyceridemia but not with low HDL-cholesterol levels in NHANES samples [12,13]. A possible explanation for the discrepancies might be that we considered each biomarker separately ( $\alpha$ - and $\gamma$-tocopherol/cholesterol ratio, respectively), whereas in the other studies vitamin $\mathrm{E}$ was defined as the sum of $\alpha$ - and $\gamma$-tocopherol. Additionally, we only had a low number of individuals $(n=37)$ with low HDL-cholesterol levels, whereas both of the other studies $[12,13]$ included more participants ( $n=4322$ and $n=8465$, respectively).

\subsubsection{Vitamin E Levels, Fatty Liver Disease, and Liver Fat Content}

The association of vitamin E levels with NASH has been assessed in some prior clinical settings with rather small samples sizes [17-19]. One study [17] reported, on average, higher serum vitamin E levels in 43 patients with histologically proven NASH as compared to 33 healthy controls. In two other studies [18,19], however, vitamin E levels were lower in biopsy-proven NASH patients $(n=50$ and $n=29$, respectively) than in controls ( $n=40$ and $n=10$, respectively).

Regarding liver fat content in postmenopausal healthy women, $\alpha$-tocopherol was identified as a predictor of MRI-determined liver fat, along with other biomarkers [35]. However, this study was based on a rather small sample $(n=48)$ of postmenopausal women, with lack of generalizability to other women and to men [35]. We expand these analyses by assessing in a much larger sample $(n=571)$ from the general population, including men and women, the associations of circulating $\alpha$ and $\gamma$-tocopherol/cholesterol ratio levels with MRI-determined LSI, a proxy for liver fat, modeled on a continuous scale and as a binary trait (FLD).

Yet, albeit FLD is commonly subdivided into non-alcoholic FLD and alcoholic FLD [36], others questioned such a distinction, e.g., because of, in part, similar pathological findings in alcoholic and non-alcoholic FLD, in part overlapping pathophysiological features, sharing of alcohol and other risk factors for FLD in a substantial fraction of the population, and a lack of a consensus regarding harmless alcohol consumption [37]. Furthermore, both non-alcoholic FLD and alcoholic FLD have been associated with premature atherosclerosis, and these findings support the paradigm that steatosis might be a precursor of an increased cardiovascular risk [38]. Therefore, considering FLD as a complex, multifactorial condition [37], we did not distinguish between alcoholic and non-alcoholic FLD but focused on MRI-derived LSI as a proxy for liver fat content.

Interestingly, vitamin E therapy (800 UI per day) for 96 weeks performed better than pioglitazone and placebo in a randomized trial in patients with NASH [6]. The primary endpoint of the study was a histological improvement of NASH features [6].

However, in contrast to the studies mentioned above, we observed no association between vitamin E levels and LSI or FLD in our sample. One potential explanation is that vitamin E levels are altered preferentially in patients with advanced liver disease [39], but not in relatively healthy men and women from the general population with rather modest alterations in liver fat, a premise that merits further investigations.

\subsection{Potential Mechanisms for the Observed Associations}

Our data suggest that circulating vitamin E ( $\alpha$ - and $\gamma$-tocopherol/cholesterol ratio) levels are positively associated with MetS and MRI-determined body fat volumes (particularly VAT).

Circulating vitamin E levels are affected by several factors: Dietary vitamin E is absorbed in the gastro-intestinal system (the efficiency of vitamin $\mathrm{E}$ absorption is widely variable, ranging from $20-80 \%$ ) and transported via chylomicrons to the liver [40]. Taken up by the liver, $\alpha$-tocopherol has several possible metabolic pathways. The hepatic $\alpha$-tocopherol transfer protein ( $\alpha$-TTP) is the major regulator for maintaining normal plasma $\alpha$-tocopherol concentrations [41]. $\gamma$-tocopherol has much less affinity $(\alpha$-tocopherol $=100 \%, \gamma$-tocopherol $=9 \%$ [42]) for $\alpha$-TTP and is largely metabolized in the 
liver and secreted in the bile [43]. Experimental evidence indicates that $\alpha$-TTP activity is modulated by oxidative stress potentially influencing vitamin E status [44-47].

$\alpha$-TTP can facilitate $\alpha$-tocopherol transfer to very low density lipoproteins (VLDL), whereas the underlying mechanism is still not understood, and facilitate its return to the liver [40]. It is suggested that VLDL is enriched with $\alpha$-tocopherol to a lesser extent in MetS compared to healthy adults and therefore transported in VLDL to a lesser degree to extrahepatic tissues in individuals with MetS because of a slower $\alpha$-tocopherol catabolism in MetS compared to healthy adults $[16,48]$. Therefore, it might be that the disappearance of $\alpha$-tocopherol from plasma is slower in individuals with MetS $[16,48]$, which would explain the positive association of plasma $\alpha$-tocopherol levels with MetS as observed in our analyses.

Looking at the excretion, $\alpha$-tocopherol can be secreted in bile for fecal excretion, but it is not known if this pathway is altered in individuals with MetS [48]. Interestingly, data from a recent study [48] indicate that MetS may inhibit the hepatic metabolism of $\alpha$-tocopherol to the $\alpha$-tocopherol metabolite $\alpha$-carboxyethyl hydroxychromanol (CEHC) (secreted in bile for elimination via feces or excreted via urine [43]). In a recent clinical trial, Traber et al. [48] observed that individuals with MetS $(n=10)$ excrete less vitamin E (lower amounts of $\alpha$ - and $\gamma$-CEHC were detected in the urine) as compared to healthy adults $(n=10)$. The authors speculated that individuals with MetS might need more vitamin $\mathrm{E}$ because of increased oxidative and inflammatory stressors, thereby suggesting they had increased requirements for $\alpha$-tocopherol and therefore retained more vitamin E compared to healthy adults [48]. We observed that $\alpha$ - and $\gamma$-tocopherol/cholesterol ratios were more strongly and positively associated with VAT than with SAT. Indeed, it is known that adipose tissue, as an endocrine organ, contains a large number of pro-inflammatory cytokines including tumor necrosis factor- $\alpha$, interleukin (IL)- $1 \beta$, and IL-6-promoting inflammatory response and oxidative stress [49,50]. Of note, VAT has been shown to release more inflammatory markers (e.g., two to three times more IL-6) than SAT [50]; a rise in concentration of inflammatory markers could be responsible for increased oxidative stress leading to higher vitamin E levels as a compensatory mechanism. Furthermore, in our study, adjustment for BMI rendered the association of $\gamma$-tocopherol/cholesterol with SAT statistically non-significant, but not the association with VAT. This might be explained by the fact that BMI is more strongly correlated with SAT than with VAT [51].

\subsection{Strengths and Limitation}

Strengths of the present study include the assessment of VAT, SAT and liver fat by MRI in a moderate-sized sample from the general population, the measurement of vitamin E in plasma, and the detailed assessment of covariates. The following limitation merits consideration. The cross-sectional study design precludes causal inferences, because exposure and outcome were assessed at the same time. Furthermore, we cannot completely rule out reverse causality. Besides, the cross-sectional study design and the small regression coefficients observed for the associations of VAT and SAT, with both $\alpha$ - and $\gamma$-tocopherol values, limit our ability to quantify and translate the observed associations into clinical meaningful findings. Moreover, we had no information about why individuals were taking vitamin E supplements and about the use of statins. However, we did have self-reported information regarding the use of lipid-lowering medications for a subsample $(n=305)$.

In summary, we observed significant associations of circulating vitamin E concentrations with MetS and MRI-determined body fat volumes (particularly VAT). Further investigations of longitudinal relationships between $\alpha$ - and $\gamma$-tocopherol levels and metabolic conditions and liver fat are warranted. 
Supplementary Materials: The following are available online at www.mdpi.com/2072-6643/9/10/1143/s1, Table S1: Missing covariates information; Table S2: Odds ratio and 95\% Confidence Interval for the association of $\alpha$-tocopherol/cholesterol ratio with individual components of MetS; Table S3: Odds ratio and 95\% Confidence Interval for the association of $\gamma$-tocopherol/cholesterol ratio with individual components of MetS; Table S4: Sensitivity analysis: multivariable-adjusted means and 95\% CI of VAT, SAT, and LSI according to tertiles of $\alpha$-tocopherol/cholesterol ratio, and scaled by IQR after excluding vitamin E supplement users; Table S5: Sensitivity analysis: multivariable-adjusted means and 95\% CI of VAT, SAT, and LSI according to tertiles of $\gamma$-tocopherol/cholesterol ratio, and scaled by IQR after excluding vitamin E supplement users; Table S6: Sensitivity analysis: odds Ratio and 95\% Confidence Interval for the association of $\alpha$ - and $\gamma$-tocopherol/cholesterol ratio with MetS and FLD after excluding vitamin E supplement users.

Acknowledgments: Manja Koch is recipient of a Postdoctoral Research Fellowship from the German Research Foundation (Deutsche Forschungsgemeinschaft, DFG, KO 5187/1-1). Romina di Giuseppe is supported by the Deutsche Forschungsgemeinschaft Excellence Cluster "Inflammation at Interfaces" (grants EXC306 and EXC306/2). The PopGen 2.0 Network is supported by the German Federal Ministry of Education and Research (grant 01GR0468 and 01EY1103). The founding sponsors had no role in the design of the study; in the collection, analyses, or interpretation of data; in the writing of the manuscript, and in the decision to publish the results.

Author Contributions: W.L. and G.R. formulated the research question; W.L., S.S. and U.N. designed the study; T.E. performed the vitamin E measurements, S.W. and R.d.G. performed the statistical analyses, S.W., W.L., R.D.G., S.P.D., M.K., I.R. and S.S. contributed to the interpretation of the data; S.W. and W.L. drafted the manuscript. All authors (S.W., R.d.G., S.P.D., T.E., M.K., S.S., I.R., M.B., J.B., J.K., H.-P. M., G.J., U.N., G.R. and W.L.) critically reviewed and approved the final version of the manuscript.

Conflicts of Interest: The authors declare no conflict of interest.

\section{References}

1. Roberts, C.K.; Sindhu, K.K. Oxidative stress and metabolic syndrome. Life Sci. 2009, 84, 705-712. [CrossRef] [PubMed]

2. Bonomini, F.; Rodella, L.F.; Rezzani, R. Metabolic syndrome, aging and involvement of oxidative stress. Aging Dis. 2015, 6, 109-120. [CrossRef] [PubMed]

3. Polimeni, L.; Del Ben, M.; Baratta, F.; Perri, L.; Albanese, F.; Pastori, D.; Violi, F.; Angelico, F. Oxidative stress: New insights on the association of non-alcoholic fatty liver disease and atherosclerosis. World J. Hepatol. 2015, 7, 1325-1336. [CrossRef] [PubMed]

4. Jiang, Q. Natural forms of vitamin E: Metabolism, antioxidant, and anti-inflammatory activities and their role in disease prevention and therapy. Free Radic. Biol. Med. 2014, 72, 76-90. [CrossRef] [PubMed]

5. Borel, P.; Preveraud, D.; Desmarchelier, C. Bioavailability of vitamin E in humans: An update. Nutr. Rev. 2013, 71, 319-331. [CrossRef] [PubMed]

6. Sanyal, A.J.; Chalasani, N.; Kowdley, K.V.; McCullough, A.; Diehl, A.M.; Bass, N.M.; Neuschwander-Tetri, B.A.; Lavine, J.E.; Tonascia, J.; Unalp, A.; et al. Pioglitazone, vitamin E, or placebo for nonalcoholic steatohepatitis. $N$. Engl. J. Med. 2010, 362, 1675-1685. [CrossRef] [PubMed]

7. Kabat, G.C.; Heo, M.; Ochs-Balcom, H.M.; LeBoff, M.S.; Mossavar-Rahmani, Y.; Adams-Campbell, L.L.; Nassir, R.; Ard, J.; Zaslavsky, O.; Rohan, T.E. Longitudinal association of measures of adiposity with serum antioxidant concentrations in postmenopausal women. Eur. J. Clin. Nutr. 2016, 70, 47-53. [CrossRef] [PubMed]

8. Chai, W.; Conroy, S.M.; Maskarinec, G.; Franke, A.A.; Pagano, I.S.; Cooney, R.V. Associations between obesity and serum lipid-soluble micronutrients among premenopausal women. Nutr. Res. 2010, 30, 227-232. [CrossRef] [PubMed]

9. Wallstrom, P.; Wirfalt, E.; Lahmann, P.H.; Gullberg, B.; Janzon, L.; Berglund, G. Serum concentrations of beta-carotene and alpha-tocopherol are associated with diet, smoking, and general and central adiposity. Am. J. Clin. Nutr. 2001, 73, 777-785. [PubMed]

10. Garcia, O.P.; Ronquillo, D.; Caamano Mdel, C.; Camacho, M.; Long, K.Z.; Rosado, J.L. Zinc, vitamin A, and vitamin $\mathrm{C}$ status are associated with leptin concentrations and obesity in Mexican women: Results from a cross-sectional study. Nutr. Metab. 2012, 9, 59. [CrossRef] [PubMed]

11. Galan, P.; Viteri, F.E.; Bertrais, S.; Czernichow, S.; Faure, H.; Arnaud, J.; Ruffieux, D.; Chenal, S.; Arnault, N.; Favier, A.; et al. Serum concentrations of beta-carotene, vitamins $C$ and $E$, zinc and selenium are influenced by sex, age, diet, smoking status, alcohol consumption and corpulence in a general French adult population. Eur. J. Clin. Nutr. 2005, 59, 1181-1190. [CrossRef] [PubMed] 
12. Beydoun, M.A.; Shroff, M.R.; Chen, X.; Beydoun, H.A.; Wang, Y.; Zonderman, A.B. Serum antioxidant status is associated with metabolic syndrome among U.S. Adults in recent national surveys. J. Nutr. 2011, 141, 903-913. [CrossRef] [PubMed]

13. Ford, E.S.; Mokdad, A.H.; Giles, W.H.; Brown, D.W. The metabolic syndrome and antioxidant concentrations: Findings from the third national health and nutrition examination survey. Diabetes 2003, 52, 2346-2352. [CrossRef] [PubMed]

14. Godala, M.M.; Materek-Kusmierkiewicz, I.; Moczulski, D.; Rutkowski, M.; Szatko, F.; Gaszynska, E.; Tokarski, S.; Kowalski, J. Lower plasma levels of antioxidant vitamins in patients with metabolic syndrome: A case control study. Adv. Clin. Exp. Med. 2016, 25, 689-700. [CrossRef] [PubMed]

15. Li, Y.; Guo, H.; Wu, M.; Liu, M. Serum and dietary antioxidant status is associated with lower prevalence of the metabolic syndrome in a study in Shanghai, China. Asia Pac. J. Clin. Nutr. 2013, 22, 60-68. [PubMed]

16. Mah, E.; Sapper, T.N.; Chitchumroonchokchai, C.; Failla, M.L.; Schill, K.E.; Clinton, S.K.; Bobe, G.; Traber, M.G.; Bruno, R.S. Alpha-tocopherol bioavailability is lower in adults with metabolic syndrome regardless of dairy fat co-ingestion: A randomized, double-blind, crossover trial. Am. J. Clin. Nutr. 2015, 102, 1070-1080. [CrossRef] [PubMed]

17. Machado, M.V.; Ravasco, P.; Jesus, L.; Marques-Vidal, P.; Oliveira, C.R.; Proenca, T.; Baldeiras, I.; Camilo, M.E.; Cortez-Pinto, H. Blood oxidative stress markers in non-alcoholic steatohepatitis and how it correlates with diet. Scand. J. Gastroenterol. 2008, 43, 95-102. [CrossRef] [PubMed]

18. Erhardt, A.; Stahl, W.; Sies, H.; Lirussi, F.; Donner, A.; Haussinger, D. Plasma levels of vitamin E and carotenoids are decreased in patients with nonalcoholic steatohepatitis (NASH). Eur. J. Med. Res. 2011, 16, 76-78. [CrossRef] [PubMed]

19. Bahcecioglu, I.H.; Yalniz, M.; Ilhan, N.; Ataseven, H.; Ozercan, I.H. Levels of serum vitamin A, alpha-tocopherol and malondialdehyde in patients with non-alcoholic steatohepatitis: Relationship with histopathologic severity. Int. J. Clin. Pract. 2005, 59, 318-323. [CrossRef] [PubMed]

20. Nothlings, U.; Krawczak, M. PopGen. A population-based biobank with prospective follow-up of a control group. Bundesgesundhbl. Gesundheitsforsch. Gesundheitsschutz 2012, 55, 831-835.

21. Krawczak, M.; Nikolaus, S.; Von Eberstein, H.; Croucher, P.J.; El Mokhtari, N.E.; Schreiber, S. Popgen: Population-based recruitment of patients and controls for the analysis of complex genotype-phenotype relationships. Community Genet. 2006, 9, 55-61. [CrossRef] [PubMed]

22. Barbaresko, J.; Siegert, S.; Koch, M.; Aits, I.; Lieb, W.; Nikolaus, S.; Laudes, M.; Jacobs, G.; Nothlings, U. Comparison of two exploratory dietary patterns in association with the metabolic syndrome in a northern German population. Br. J. Nutr. 2014, 112, 1364-1372. [CrossRef] [PubMed]

23. Nothlings, U.; Hoffmann, K.; Bergmann, M.M.; Boeing, H. Fitting portion sizes in a self-administered food frequency questionnaire. J. Nutr. 2007, 137, 2781-2786. [PubMed]

24. Dehne, L.I.; Klemm, C.; Henseler, G.; Hermann-Kunz, E. The German food code and nutrient data base (BLS II.2). Eur. J. Epidemiol. 1999, 15, 355-359. [CrossRef] [PubMed]

25. Muller, H.P.; Raudies, F.; Unrath, A.; Neumann, H.; Ludolph, A.C.; Kassubek, J. Quantification of human body fat tissue percentage by MRI. NMR Biomed. 2011, 24, 17-24. [CrossRef] [PubMed]

26. Fischer, K.; Moewes, D.; Koch, M.; Muller, H.P.; Jacobs, G.; Kassubek, J.; Lieb, W.; Nothlings, U. Mri-determined total volumes of visceral and subcutaneous abdominal and trunk adipose tissue are differentially and sex-dependently associated with patterns of estimated usual nutrient intake in a northern German population. Am. J. Clin. Nutr. 2015, 101, 794-807. [CrossRef] [PubMed]

27. Koch, M.; Borggrefe, J.; Barbaresko, J.; Groth, G.; Jacobs, G.; Siegert, S.; Lieb, W.; Muller, M.J.; Bosy-Westphal, A.; Heller, M.; et al. Dietary patterns associated with magnetic resonance imaging-determined liver fat content in a general population study. Am. J. Clin. Nutr. 2014, 99, 369-377. [CrossRef] [PubMed]

28. Alberti, K.G.; Eckel, R.H.; Grundy, S.M.; Zimmet, P.Z.; Cleeman, J.I.; Donato, K.A.; Fruchart, J.C.; James, W.P.; Loria, C.M.; Smith, S.C., Jr.; et al. Harmonizing the metabolic syndrome: A joint interim statement of the international diabetes federation task force on epidemiology and prevention; national heart, lung, and blood institute; American heart association; World heart federation; International atherosclerosis society; and International association for the study of obesity. Circulation 2009, 120, 1640-1645. [PubMed] 
29. Szczepaniak, L.S.; Nurenberg, P.; Leonard, D.; Browning, J.D.; Reingold, J.S.; Grundy, S.; Hobbs, H.H.; Dobbins, R.L. Magnetic resonance spectroscopy to measure hepatic triglyceride content: Prevalence of hepatic steatosis in the general population. Am. J. Physiol. Endocrinol. Metab. 2005, 288, E462-E468. [CrossRef] [PubMed]

30. Haftenberger, M.; Schuit, A.J.; Tormo, M.J.; Boeing, H.; Wareham, N.; Bueno-de-Mesquita, H.B.; Kumle, M.; Hjartaker, A.; Chirlaque, M.D.; Ardanaz, E.; et al. Physical activity of subjects aged 50-64 years involved in the European prospective investigation into cancer and nutrition (EPIC). Public Health Nutr. 2002, 5, 1163-1176. [CrossRef] [PubMed]

31. Ainsworth, B.E.; Haskell, W.L.; Herrmann, S.D.; Meckes, N.; Bassett, D.R., Jr.; Tudor-Locke, C.; Greer, J.L.; Vezina, J.; Whitt-Glover, M.C.; Leon, A.S. 2011 compendium of physical activities: A second update of codes and MET values. Med. Sci. Sports Exerc. 2011, 43, 1575-1581. [CrossRef] [PubMed]

32. Augustin, K.; Blank, R.; Boesch-Saadatmandi, C.; Frank, J.; Wolffram, S.; Rimbach, G. Dietary green tea polyphenols do not affect vitamin E status, antioxidant capacity and meat quality of growing pigs. J. Anim. Physiol. Anim. Nutr. 2008, 92, 705-711. [CrossRef] [PubMed]

33. Kayden, H.J.; Traber, M.G. Absorption, lipoprotein transport, and regulation of plasma concentrations of vitamin E in humans. J. Lipid Res. 1993, 34, 343-358. [PubMed]

34. Thurnham, D.I.; Davies, J.A.; Crump, B.J.; Situnayake, R.D.; Davis, M. The use of different lipids to express serum tocopherol: Lipid ratios for the measurement of vitamin E status. Ann. Clin. Biochem. 1986, 23, 514-520. [CrossRef] [PubMed]

35. Lim, U.; Turner, S.D.; Franke, A.A.; Cooney, R.V.; Wilkens, L.R.; Ernst, T.; Albright, C.L.; Novotny, R.; Chang, L.; Kolonel, L.N.; et al. Predicting total, abdominal, visceral and hepatic adiposity with circulating biomarkers in Caucasian and Japanese American women. PLoS ONE 2012, 7, e43502. [CrossRef] [PubMed]

36. Italian Association for the Study of the Liver. AISF position paper on nonalcoholic fatty liver disease (NAFLD): Updates and future directions. Dig. Liver Dis. 2017, 49, 471-483.

37. Volzke, H. Multicausality in fatty liver disease: Is there a rationale to distinguish between alcoholic and non-alcoholic origin? World J. Gastroenterol. 2012, 18, 3492-3501. [CrossRef] [PubMed]

38. Loria, P.; Marchesini, G.; Nascimbeni, F.; Ballestri, S.; Maurantonio, M.; Carubbi, F.; Ratziu, V.; Lonardo, A. Cardiovascular risk, lipidemic phenotype and steatosis. A comparative analysis of cirrhotic and non-cirrhotic liver disease due to varying etiology. Atherosclerosis 2014, 232, 99-109. [CrossRef] [PubMed]

39. Pacana, T.; Sanyal, A.J. Vitamin E and nonalcoholic fatty liver disease. Curr. Opin. Clin. Nutr. Metab. Care 2012, 15, 641-648. [CrossRef] [PubMed]

40. Schmolz, L.; Birringer, M.; Lorkowski, S.; Wallert, M. Complexity of vitamin E metabolism. World J. Biol. Chem. 2016, 7, 14-43. [CrossRef] [PubMed]

41. Traber, M.G. Vitamin E regulatory mechanisms. Annu. Rev. Nutr. 2007, 27, 347-362. [CrossRef] [PubMed]

42. Hosomi, A.; Arita, M.; Sato, Y.; Kiyose, C.; Ueda, T.; Igarashi, O.; Arai, H.; Inoue, K. Affinity for alpha-tocopherol transfer protein as a determinant of the biological activities of vitamin $\mathrm{E}$ analogs. FEBS Lett. 1997, 409, 105-108. [CrossRef]

43. Traber, M.G. Mechanisms for the prevention of vitamin E excess. J. Lipid Res. 2013, 54, 2295-2306. [CrossRef] [PubMed]

44. Etzl, R.P.; Vrekoussis, T.; Kuhn, C.; Schulze, S.; Poschl, J.M.; Makrigiannakis, A.; Jeschke, U.; Rotzoll, D.E. Oxidative stress stimulates alpha-tocopherol transfer protein in human trophoblast tumor cells bewo. J. Perinat. Med. 2012, 40, 373-378. [CrossRef] [PubMed]

45. Ulatowski, L.; Dreussi, C.; Noy, N.; Barnholtz-Sloan, J.; Klein, E.; Manor, D. Expression of the alpha-tocopherol transfer protein gene is regulated by oxidative stress and common single-nucleotide polymorphisms. Free Radic. Biol. Med. 2012, 53, 2318-2326. [CrossRef] [PubMed]

46. Usenko, C.Y.; Harper, S.L.; Tanguay, R.L. Fullerene C60 exposure elicits an oxidative stress response in embryonic zebrafish. Toxicol. Appl. Pharmacol. 2008, 229, 44-55. [CrossRef] [PubMed]

47. Otulakowski, G.; Engelberts, D.; Arima, H.; Hirate, H.; Bayir, H.; Post, M.; Kavanagh, B.P. Alpha-tocopherol transfer protein mediates protective hypercapnia in murine ventilator-induced lung injury. Thorax 2017, 72, 538-549. [CrossRef] [PubMed]

48. Traber, M.G.; Mah, E.; Leonard, S.W.; Bobe, G.; Bruno, R.S. Metabolic syndrome increases dietary alpha-tocopherol requirements as assessed using urinary and plasma vitamin E catabolites: A double-blind, crossover clinical trial. Am. J. Clin. Nutr. 2017, 105, 571-579. [CrossRef] [PubMed] 
49. Marseglia, L.; Manti, S.; D’Angelo, G.; Nicotera, A.; Parisi, E.; Di Rosa, G.; Gitto, E.; Arrigo, T. Oxidative stress in obesity: A critical component in human diseases. Int. J. Mol. Sci. 2014, 16, 378-400. [CrossRef] [PubMed]

50. Fonseca-Alaniz, M.H.; Takada, J.; Alonso-Vale, M.I.; Lima, F.B. Adipose tissue as an endocrine organ: From theory to practice. J. Pediatr. 2007, 83, S192-S203. [CrossRef]

51. Camhi, S.M.; Bray, G.A.; Bouchard, C.; Greenway, F.L.; Johnson, W.D.; Newton, R.L.; Ravussin, E.; Ryan, D.H.; Smith, S.R.; Katzmarzyk, P.T. The relationship of waist circumference and BMI to visceral, subcutaneous, and total body fat: Sex and race differences. Obesity 2011, 19, 402-408. [CrossRef] [PubMed]

2017 by the authors. Licensee MDPI, Basel, Switzerland. This article is an open access article distributed under the terms and conditions of the Creative Commons Attribution (CC BY) license (http://creativecommons.org/licenses/by/4.0/). 